\title{
La disonancia cognoscitiva en la experiencia del activismo gráfico
}

\section{Cognitive Dissonance in the Experience of Graphic Activism}

\author{
Adolfo Guzmán Lechuga \\ gl_adolfo@hotmail.com \\ Universidad Autónoma de Coahuila \\ Escuela de Artes Plásticas. \\ Saltillo, Coahuila, México
}

\author{
María del Socorro \\ Gabriela Valdéz Borroel \\ gabos99@hotmail.com \\ Universidad Autónoma de Coahuila, \\ Escuela de Artes Plásticas. \\ Saltillo, Coahuila, México \\ ORCID: 0000-0002-8627-0137
}

Recibido: 21 de noviembre de 2016 Aprobado: 09 de enero de 2017 Publicado: 31 de enero de 2017
Resumen:

El texto presenta los resultados del diseño cuasiexperimental realizado con alumnos de la carrera de Diseño Gráfico en la Escuela de Artes Plásticas en Saltillo, Coahuila, México.

Mediante dicho diseño, se indaga si los alumnos que abordaron problemas sociales realizando activismo gráfico desarrollaron una disonancia cognoscitiva y si experimentaron un cambio de actitud. De igual modo, el texto expone los resultados cualitativos acerca de las facilidades, dificultades y aprendizajes que los estudiantes experimentaron.
Abstract:

This paper presents the results of a quasi-experimental design made with the students of the Graphic Design Undergraduate Degree Program in the Visual Arts School on Saltillo, Coahuila, Mexico.

Through this design we look into whether the students who touched on social problems in their graphic activism developed cognitive dissonance and if they showed any change of attitudes. Likewise, the paper presents the qualitative results on the ease and difficulties and lessons that the students had.

Palabras clave: disonancia cognoscitiva, activismo gráfico. Keywords: cognitive dissonance, graphic activism. 


\section{Introducción}

ic arlos Muñoz, siguiendo a Harry Trandis (1971), sostiene que los problemas importantes del siglo XX están relacionados con actitudes. Dice que los países ricos (Alemania, Canadá, Estados Unidos, Reino Unido, Japón, Kuwait, Hong Kong) se vuelven más ricos mientras que los pobres se hacen más pobres. Esto sucede dentro de un mundo que parece hacerse más pequeño. Es así como Trandis, afirma que la humanidad tiene los conocimientos técnicos para hacer los cambios necesarios en el mundo. Sin embargo, la mayoría de los seres humanos no posee las actitudes necesarias para hacerlos (Salazar, et al., 2003:168).

En la actualidad, los individuos que ingresan al sistema educativo superior interpretan la libertad como el derecho a hacer lo que deseen. Tal actitud puede formar parte del perfil de un alumno inconsciente, con poca responsabilidad de sus actos y sin interés por lo que sucede en su ambiente socia. Estos estudiantes no consideran que su desinterés tiene consecuencias en el entorno y dichas consecuencias pueden, a su vez, producir situaciones negativas que los afecten. Así, muchos estudiantes viven ajenos e indiferentes a los problemas sociales que los rodean, sin participar con ideas ni acciones para resolverlos en su comunidad.

El entorno social actual es un tanto complicado y aunado a las dificultades de convivencia, tolerancia existe la falta de interés de los alumnos de nivel licenciatura por participar activamente en él. Una de las razones por lo que esto ocurre podría ser, la poca atención que en la educación de las nuevas generaciones se pone en la información y la participación de los alumnos respecto de los problemas sociales y del conocimiento de su comunidad ${ }^{1}$. Esto se ve reflejado en la inconsciencia e indiferencia por parte de los alumnos que egresan del sistema de educación superior pública y que se incorporan al ámbito laboral local.

Quizás la respuesta a los problemas sociales añejos, el retroceso en la convivencia y hasta la deshumanización creciente, encuentran su causa en la falta de actitudes positivas como las de interés, compromiso y responsabilidad por nuestro entorno social y ambiental. Lo ha dicho Trandis, al sugerir que poseemos los conocimientos técnicos, pero no tenemos las actitudes. Ante ello, surge la pregunta central: ¿Cómo hacer para que las personas desarrollen las actitudes que hagan la diferencia? Una posible respuesta puede encontrarse en los procesos de formación en las universidades. Es en estas instituciones donde puede existir la posibilidad más prometedora para desarrollarlas. Como centros formativos pueden propiciar 
experiencias en los alumnos en las que desarrollen las actitudes de interés y compromiso por el entorno social. La oportunidad para las experiencias puede estar en combinación con el activismo gráfico, actividad que "induce, persuade y convoca a la participación activa de la sociedad en temas sociales, políticos y del medio ambiente" (Guzmán, A., 2014:133) con propuestas gráficas que salen de lo común. Y sobre todo porque el activismo gráfico tiene como "propósito lograr la concientización de los individuos sobre los temas sociales y del ambiente en donde se pretende hacer un cambio social" (Guzmán, A., 2014:133).

Es así como consideramos importante desarrollar un diseño cuasiexperimental ${ }^{2}$ que demuestre dicha suposición y del que cabe precisar, es un derivado del diseño experimental y que según Roberto Hernández "manipula deliberadamente al menos una variable independiente para ver su efecto con una o más variables dependientes" (Hernández, R. et al., 1998:169). Esta clase de pruebas se diferencian de los experimentos llamados "verdaderos", solo en el grado de seguridad y confiabilidad que se puede lograr sobre la equivalencia de los grupos, de tal modo que los cuasiexperimentos son muy parecidos a los experimentos "verdaderos".

El experimento también tiene el interés de mostrar lo fundamental que es proporcionar información al alumno sobre los problemas sociales durante y para el proceso de educación, ya que permite conocer y vivir experiencias que lo facultan para encarar la realidad, eliminando así la indiferencia y forjando alumnos activos y participativos, con valores, ideología, creencias, tendencia política, preferencias, en fin una amplia cantidad de agentes esenciales en su formación y que son determinantes para su actuar profesional.

Actitudes y conducta
Julio Villegas (2003) de acuerdo con Allport (1935) afirma que la actitud es "un estado mental y neural de la disposición a responder, organizada a través de la experiencia y que ejerce una influencia directiva y/o dinámica de la conducta" (Salazar, J., et al., 2003:148). En este sentido, la adopción o cambio de actitud estará definida por la postura que se adopta y la reacción con que una persona responde ante un objeto o hecho, de acuerdo a la importancia y experiencia vivida. En este sentido Fishbein (1967) menciona que la actitud es "la predisposición aprendida para responder ante un objeto de un modo consistentemente favorable o desfavorable" (Salazar, J., et al., 2003:148). En ese mismo tenor Warren (1979:4) considera que la actitud es una "disposición o preparación estabilizada. Experiencia abreviada pero amplia"³. Es así como

\footnotetext{
${ }^{1}$ El sistema educativo profesional público no esta ofreciendo suficientes posibilidades de relacionarse con la realidad social. Las instituciones solo se llevan acabo algunos eventos de moda que no implican mayor compromiso y responsabilidad. De ahí que no exista progreso en el crecimiento de la actitud de interés y conocimiento en el alumno sobre las dificultades comunitarias. Opinión de los autores con experiencia de dieciocho años en la docencia pública y privada.

2 Según Hernándes Sampieri en los diseños cuasiexperimentales "los sujetos no son asignados al azar a los grupos ni emparejados; sino que dichos grupos ya estaban formados antes del experimento, son grupos intactos". Por ejemplo, los grupos escolares utilizados para este trabajo, los cuales ya estaban formados con anterioridad al experimento. Hernández, R., Fernández, C., Baptista, P. (1998). Metodología de la investigación. México., Mc Graw Hill., p. 169.
} 
sostenemos que la actitud es la preparación psíquica y hasta corporal para vivir una experiencia que nace y en la que se espera una repercusión de pensamiento. Por ejemplo, como sugerir ponerse en actitud, al estar en disposición para recibir la experiencia sin oponer resistencia. Por ello, es imperativo para la formación de actitudes romper con las barreras ideologicas de las personas, porque éstas son las que coartan la posibilidad de vivir la experiencia que genera un punto de vista nuevo al considerar encontrarse con experiencias físicas e ideologicas frente a objetos, estímulos o procesos.

En todo caso, actitud también se considera el estar con una apertura ideológica para recibir las experiencias. Es así que podemos hablar del desarrollo de actitudes sociales, que se definen como el conjunto de "actitudes de un individuo que están específicamente dirigidas hacia otros miembros de la especie, o que han sido desarrolladas en la intención social" (Warren, 1979:4). Por tanto, un elemento determinante en la formación de actitudes sociales son las experiencias con las que se aprenden posturas y disposiciones para actuar en relación con los otros, siendo el interés en el cómo se aprende y cómo se da el aprendizaje social. Entonces la formación de actitudes de tipo social es a través del diseño de tareas que vinculan al alumno con situaciones reales, es decir, aproximandolo a los problemas de su entorno. En este sentido, sin lugar a duda las actividades educativas relacionadas con los problemas son la estrategia de mayor utilidad, sobre todo cuando lo individuos son reacios a revelar sus verdaderas actitudes o las desconocen.

Componentes de las actitudes
Si la actitud es la disposición para actuar, ¿cuales son los elementos que definen ese actuar? Entre los especialistas existen distintas definiciones y componentes que ponen la definición de éste asunto en un estado de controversia. A nuestro parecer y apoyados en la definición inicial de Allport, estimamos que la más acertada es la que considera la parte afectiva, cognitiva y conductual. A ese respecto, Zimbardo y Ebbesen (1970) en Salazar et al. (2003) argumentan que el componente afectivo es la evaluación de la respuesta emocional (si gusta o disgusta una persona u objeto), el cognitivo son las creencias y conocimiento fatual que el individuo tiene sobre una persona u objeto, mientras que el componente conductual es el proceder dirigido a la persona u objeto (Salazar et al., 2003:155). Estos son los tres elementos que describen con amplitud la naturaleza de las actitudes que la persona utiliza para evaluar y en consecuencia actuar.

\footnotetext{
${ }^{3}$ Existen mediciones fisiológicas para la actitud que se realizan directamente, midiendo las reacciones emocionales que se producen ante la presencia de un objeto de actitud. Como lo son el ritmo cardiaco, descarga de adrenalina, etc. Sin embargo, estas mediciones son limitadas para ser consideradas con suficiente inferencia sobre las actitudes
} 
Vinculación entre actitud y conducta

La falta de coherencia entre el pensar y el actuar de una persona es un asunto cotidiano. Parcialmente se actua cómo se piensa. Lo común es decir una cosa y hacer otra, esto es, ser inconsistente entre el pensar y el actuar. Algunos especialistas cómo Fishbein (1967), apoyados en experimentos sugieren que conocer la actitud de un individuo permite conocer su conducta. Sin embargo, lo que parece tener mayor sustento es el hecho de que las personas "realizan" sus actitudes a partir de su comportamiento. De tal manera que la actitud no ejerce una acción directa sobre la conducta, y el conocimiento de las actitudes no son garantia para predecir las conductas. También es verdad que el trabajar para formar actitudes (por las dificultades sociales) no garantiza nuevas conductas. Sin embargo, lo que se pretende en esta indagación, es propiciar un nuevo punto de vista en el alumno, desarrollando en él una postura de interés y disposición a partir de la información con la que se experimenta.

Recordemos que la actitud se forma con la experiencia, creencias y conocimiento. Siendo que las experiencias se valoran por su aplicación en situaciones que son repetitivas o parecidas a eventos pasados y como un conocimiento que se alberga y es valorado por su utilidad. Pero también es cierto que la experiencia guarda cierto conocimiento al cual se le da valor negativo o mala experiencia. De este modo, se espera que las experiencias "positivas y negativas" que viva el alumno con la realidad social, se conviertan en un conocimiento útil y valorado.

La actitud está determinada por las experiencias vividas, y es con nuevas experiencias que las actitudes pueden ser cambiadas y modificadas. El cambio de actitud tiene su origen en el nuevo aprendizaje, el cual para alcanzar el cambio debe ser reforzado. Mc Guire (1969) considera "al refuerzo cómo un factor crítico en el proceso de cambio actitudinal. Si el individuo recibe refuerzo, la respuesta se fortalece; si, por el contrario, no se recibe ese refuerzo o se castiga, la respuesta se extingue" (Salazar et al., 2003:171).

Teoría de la disonancia cognoscitiva
La teoría de la disonancia cognoscitiva de Leon Festinger (1957) está enmarcada en el campo de las teorías del aprendizaje social. Festinger supone que la formación de actitudes es "la búsqueda y mantenimiento de la consistencia (equilibrio) entre varios elementos de la estructura cognoscitiva" (Salazar et al., 2003:174).

Es así como en esta perspectiva de aprendizaje, el diseño del cuasiexperimento de disonancia cognocitiva en la experiencia del activismo gráfico busca mostrar evidencia acerca de cómo al insertar los temas del activismo gráfico se altera la armonía interna del alumno, experimentando una disonancia que lo inicia en la formación o modificación de actitudes, sobre todo las de interés, compromiso y responsabilidad por lo que sucede en su entorno social.

Los temas del activismo gráfico: protección animal, violencia física y psicológica, justicia e igualdad social, medio ambiental, la paz, libertad de expresión, censura y defensa de la infancia, son el detonante de una inconsistencia en los elementos 
"afectivos, cognoscitivos o conductuales de una actitud, o entre dos elementos cognoscitivos o cualquier otro tipo de inconsistencia cognoscitiva" (Salazar et al., 2003:174).

En esta situación el alumno experimenta un estado nada confortable (perturbador) que le genera incomodidad y en donde no hay placer. Para reducir esos estados, hará lo necesario, ocupándose de la realidad o probablemente deformándola.

Según Festinger, un estado o situación de disonancia es aquel que dirige al organismo en una dirección específica la cual puede buscar reducir la tensión provocada por el hecho o evitar que la incomodidad siga en aumento (Salazar et al., 2003:175). De esta manera es que la disonancia provocada en el alumno con la producción de activismo gráfico hará que las experiencias, percepciones y conocimientos se alteren y resulten en "cambios de conducta, cambios cognoscitivos o una exposición circunspecta hacia nueva información que pueda estar produciendo alteración" (Salazar et al., 2003:175-176), como lo es la perturbación provocada con la realidad que les presenta el activismo gráfico, sea por lo desconocido de los temas o la relevancia que le da cada alumno. Lo cierto es que con cualquiera de estas variables se provoca en el alumno una disonancia, que le hace adoptar una dirección para sus pensamientos y acciones. Así lo ha sugerido Festiger, al distinguir las tres variables conceptuales que producen mayor o menor disonancia:

a) La importancia que tenga cada uno de los elementos cognoscitivos.

b) El número de elementos cognoscitivos consonantes y disonantes que existan simultáneamente. Esto es, que mientras mayor sea el número de elementos disonantes en relación a elementos consonantes, mayor será la disonancia que el individuo experimenta.

c) La llamada "sobreposición cognoscitiva". Es decir, la semejanza entre dos elementos correspondientes a dos alternativas diferentes. La equivalencia funcional de los objetos o actividades representadas por las cogniciones va a influir en la cantidad o magnitud de la disonancia que se experimenta. Cuanto mayor sea el grado de sobreposición cognoscitiva, menor va a ser la disonancia resultante (Salazar et al., 2003:176).

Para Festinger, estas variables proponen dos hipótesis: La primera, es que ante una disonancia que psicológicamente causa incomodidad, la persona busca reducirla para lograr consonancia. La segunda, es que para cuando hay disonancia, la persona intentará reducirla evitando situaciones e información que la aumente.

El cuasiexperimento realizado localiza evidencia de comprobación para ambas hipótesis. Aunque con mayor peso en la primera de ellas, en donde la información y producción del activismo gráfico provocó en los alumnos una incomodidad psicológica, al buscar reducirla a través de la documentación, comprensión e interés en los temas. Mientras que en la segunda hipótesis algunos temas como la homosexualidad, el machismo y la violencia psicológica fueron evadidos sin profundizar en la información, ya que representaban un cuestionamiento hacia la visión el manejo en su vida social. 
Los resultados obtenidos a través de la expresión oral, escrita y de producción gráficos son evidencia que confirma lo dicho por Festinger, respecto a que "la disonancia, o sea la existencia de relaciones entre cogniciones que no concuerdan, son un factor de la motivación, y lo es por derecho propio" (Salazar et al., 2003:176).

La disonancia es una situación que podemos experimentar todos los días. Un alumno entra en estado de disonancia cuando le llega información nueva, haciendo discordancia con la información que conoce o con el conocimiento de una conducta determinada. Festinger afirma que casi la totalidad de acciones que emprende una persona o por cada sentimiento que tiene existen altas probabilidades para que por lo menos un elemento cognoscitivo genere un estado de disonancia (Ovejero, 1975:205). Es así como cada encuentro con eventos o ideas pueden generar una disonancia de cierta magnitud, siendo lo importante hacia dónde y cómo dirigir los efectos de la disonancia. Ante tal situación en igualdad de proporción se dispara una respuesta que pretende estabilizar o reducir el hecho, ya que el alumno requiere sentirse en coherencia. Ante esa disonancia intentará establecer una armonía interna consiguiendo "consistencia o congruencia entre sus actitudes, opiniones, conocimientos y valores. Es decir, una tendencia hacia la consonancia, entre cogniciones" (Ovejero, 1975:205).

Método La muestra para realizar el cuasiexperimento fue de 100 alumnos en la Escuela de Artes Plásticas Rubén Herrera de Saltillo, Coahuila. Se seleccionó a tres grupos de alumnos de diferentes cursos y se les informó cuáles eran los temas que aborda el activismo gráfico: protección animal, violencia física y psicológica, justicia e igualdad social, medio ambiental, la paz, libertad de expresión, censura y defensa de la infancia. Aunado a ello se proyectaron videos informativos para cada uno de estos temas.

A cada alumno se le asignaron tres temas para desarrollar propuestas gráficas usando palabras y gráficos reconocibles, además de un eslogan, marcas o productos existentes para descontextualizar, hacer crítica, sátira, burla, ironía, denuncia o invitación. Al final, respondieron un cuestionario de opinión sobre la experiencia.

Resultados Aspectos fáciles y difíciles de gestionar para los alumnos en la experiencia del activismo gráfico. La muestra de 100 alumnos de la EAP que participaron en el cuasiexperimento y que produjeron activismo gráfico, manifiestan haber tenido dificultad de afrontar y tratar los temas, ya que eran ignorantes de éstos y debían buscar la información para abordarlos de manera adecuada. Consideran los temas con gran valor, por no relacionarse con asuntos comerciales y por tener repercusión a nivel personal al cuestionar la consciencia. Según testimonios de la mayoría 
de estudiantes, fue una experiencia satisfactoria, enriquecedora y de aprendizaje sobre el entorno social. La generalidad coincide en que la experiencia propicia el interés en los problemas sociales. Están convencidos que el activismo gráfico puede influir en la opinión de las personas de manera positiva haciendo que comprendan y conozcan el panorama social en que vivimos, comenzando por ellos mismos. Dicen que la experiencia los llevó a reflexionar y mejorar como personas a nivel de sensaciones y emociones. Dicen que la experiencia les provocó una discordancia al conocer la realidad y confrontarla con lo que conocían y sabían. Así también opinaron que informarse sobre los temas sociales desarrolla las actitudes positivas de colaboración e interés por otros individuos de su comunidad.

Algunos estudiantes expresaron que es "fácil" hacer diseño para una causa social porque no es un asunto comercial, y ello lo convierte en algo motivante y atrayente, les permite expresar una opinión y una cierta actitud a través de una imagen. De esta manera, el activismo gráfico da la oportunidad de diseñar con libertad al permitir utilizar cualquier recurso de expresión gráfica para comunicar, y no existir un compromiso y encargo comercial, pero sí el de la crítica social.

Otros alumnos dicen que diseñar para abordar los temas de violencia física y psicológica, defensa de la infancia e igualdad fue difícil, pues les generó una incomodidad psicológica que desequilibró sus escasos conocimientos sobre esos temas. Mencionan que es difícil exponerse a las imágenes que hay en internet, ya que se dan cuenta de una realidad que prácticamente les era desconocida. Para otros, la experiencia representó un cuestionamiento a su conducta puesto que, en algún momento de su vida, sin ser conscientes, habían contribuido a agravar algún problema social, como por ejemplo en el caso de la desigualdad o la violencia. Algunas otras complicaciones expresadas fueron asimilar y procesar la información de modo que, al proyectar un mensaje, éste no generara rechazo u ofendiera al espectador, pero que al mismo tiempo mostrara cierta innovación e impacto. También fue un tanto complicado lograr representaciones gráficas que hicieran consciencia y al mostraran una postura crítica.

Aportaciones para el individuo que realiza activismo gráfico
Después de trabajar en el proyecto, algunos estudiantes modificaron alguna actitud, ya sea el valor para expresar que viven de cerca los problemas abordados durante el proceso, o para decir que los viven en carne propia. Por ejemplo, aquellos que dicen tener amigos o familiares que son homosexuales y saben que han sido discriminados o despreciados incluso por ellos mismos.

El impacto fue tal que desarrollaron empatía y decidieron adherirse a alguna causa. Un alumno comentó su experiencia sobre el tema de la defensa infantil: "Aprendí a defender como me sea posible a los que no pueden hacerlo y a tratar por igual a las personas sin ver quiénes son o cómo son." Otra opinión sobre la experiencia fue que participar, lo llevó a reflexionar sobre aquellos profesionales que hacen diseño sin fundamento, que no consideran al contexto y tampoco al usuario, siendo que podrían producir diseño que forme consciencia y ayude a las personas a mejorar 
su relación con otros seres humanos. Por lo dicho, se puede suponer que realizar activismo gráfico aporta empatía y desarrolla el sentido humano.

Otros alumnos se identificaron con la lucha contra el maltrato animal y decidieron cambiar su estilo de vida al ya no comer carne. Otros expresaron que cambiarían su actitud al reconocerse como machistas o controladores en sus relaciones personales.

Algunos llegaron a la conclusión de que el tema del maltrato infantil es un asunto fundamental en el desarrollo humano de las personas y ahora, consideran indispensable que sea revertido. En este sentido, piensan que el origen de todo esto es la violencia psicológica, como punto de partida de otros fenómenos.

Los alumnos reflexionaron acerca de cómo antes del experimento ignoraban los temas que abordaron, pero después de informarse, la indiferencia desapareció y ahora se consideran más cercanos a esa realidad. Se perciben a sí mismos más reflexivos y dispuestos, con una sensación de pertenencia y preocupación social. El encuentro con la realidad les aportó interés y motivación para hacer algo para ayudar a la sociedad participando en causas no comerciales porque en su mente hubo un cambio.

La experiencia de realizar activismo gráfico puede desarrollar en el individuo distintas sensaciones y opiniones además de la empatía, preocupación e interés; también puede producir desprecio o rechazo por determinados temas si no hay un manejo correcto de la información dentro del proceso formativo del estudiante.

Aportación del activismo gráfico a la creatividad

Activismo gráfico en la educación del diseñador gráfico
El activismo gráfico propone un nuevo campo de acción para el diseñador gráfico y es una alternativa real para propiciar el desarrollo creativo, ya que al diseñar mensajes con tema un social, se deben buscar otros caminos y formas para emitir mensajes, considerando que éstos requieren de un tratamiento distinto a los mensajes comerciales. Por ello, es posible sugerir que el conocimiento de los temas sociales puede lograr conducir al alumno a ser objetivo en la aplicación de sus conocimientos de composición, estructura y psicología del color, además de experimentar con otros medios de expresión, como el realizar bocetos de manera orgánica con distintos materiales y técnicas. La experiencia motivó la creatividad y la curiosidad para emplear otros métodos e instrumentos como nuevas fuentes de información, el proceso de bocetaje, vectorizar, ejecutar y hasta la manera de implementar las nuevas propuestas.

En la discusión sobre si el activismo gráfico debe ser parte de la formación académica del diseñador, la opinión fue polarizada. Mientras algunos consideran que es importante para la formación, otros opinan que no debiera ser estrictamente parte del aprendizaje, sino ser parte de los temas o materias opcionales. 
Aquellos que están a favor, dicen que sería adecuado incluirlos en la malla curricular como una materia de índole formal. Consideran que serían de gran ayuda estos temas en la formación del diseñador, pues éste podría aprovechar sus conocimientos de persuasión para aplicarlos en la producción de activismo gráfico y así, influir de manera positiva en la opinión de las personas sobre los problemas sociales.

Otras opiniones fueron en el sentido de que el activismo gráfico sería importante como parte de los programas formales de educación porque sensibiliza acerca de lo que ocurre en el entorno social. Un alumno refirió que: "Si fuera parte de la currícula, daría la posibilidad de estar en contacto directo con el entorno y así entender los problemas". Consideran que el contacto con los temas que toca el activismo gráfico da la oportunidad de mejorar algunos asuntos en el mundo. Dicen que, como futuros profesionistas, trabajar estos temas complementa su formación en valores, consciencia y ocupación por el bien común.

Los que consideran el activismo gráfico como un tema opcional dicen que el activismo gráfico es más que diseño, porque implica una vocación y un interés personal, por lo que sería un tanto represivo obligar al estudiante, aunque es interesante participar, pues genera inquietud por el entorno social y ambiental. Suponen que el activismo gráfico ayudaría a desarrollar su lado humanísta y trabajar de manera altruista ya que, por lo general, piensan en una remuneración al momento de realizar algún proyecto. Dicen que esta actividad se realiza como consecuencia de una decisión personal, y por ello, no debe formar parte de la currícula, sino que la opción debe estar abierta para que participe el que lo desee de manera libre, como parte de una experiencia sensibilizadora para el estudiante en donde ampliará algunas habilidades.

Relevancia de trabajar con los problemas sociales
Determinar si los temas sociales son relevantes en la educación del diseñador es parte de la discusión de esta investigación. Según algunos participantes, es de suma importancia para el desarrollo humano, mientras que, para otros, tiene poca relevancia.

Algunos opinan que el diseñador no debe limitarse a seguir la moda y a trabajar en el diseño comercial, porque pierde su sentido de responsabilidad y compromiso con la sociedad al no comunicar aquellas situaciones que laceran y deterioran al individuo y al entorno. No solo se debe diseñar para temas mercantiles, sino también para temas que mejoren nuestras conductas de vida y actitudes, contribuyendo con mensajes que contrarresten los conflictos sociales.

El diseñador debe ser una figura socialmente responsable pero no sólo en los productos gráficos que genera, sino también en su actitud hacia el entorno. Es necesario formar diseñadores comprometidos socialmente que actúen y busquen cambiar actitudes sociales anómalas. Sugieren que se debe intervenir en los problemas sociales, por tanto, es importante sensibilizar y preparar al estudiante. 
Por ello, el profesor pudiera despertar el interés por las causas sociales en el alumno y la institución educativa integrando los problemas sociales en su proyecto educativo.

\section{Muestra gráfica de resultados}

\section{Mientas tanto en otra realidad...}
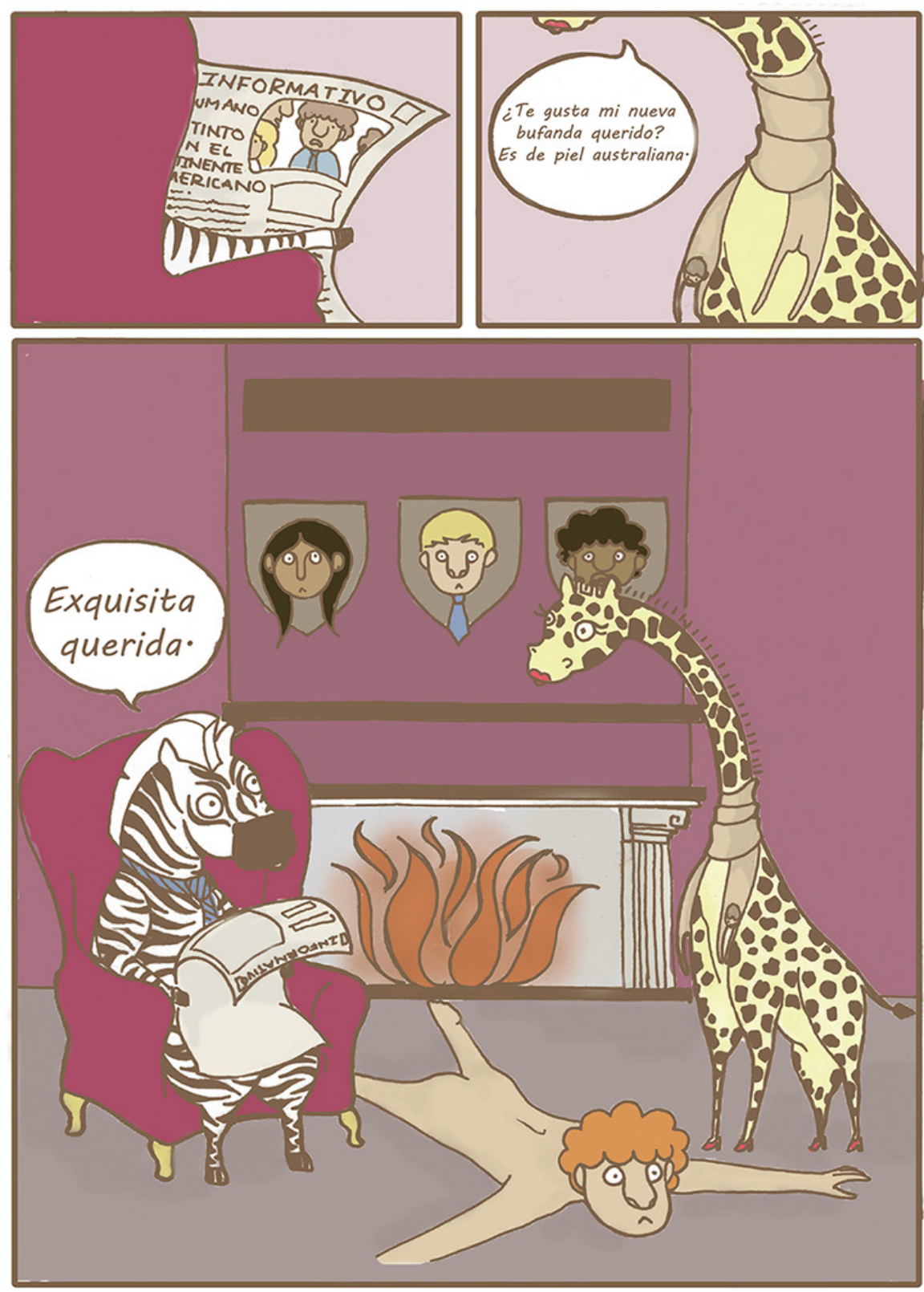

Fig.1 Protección animal - Catalina Elizalde 


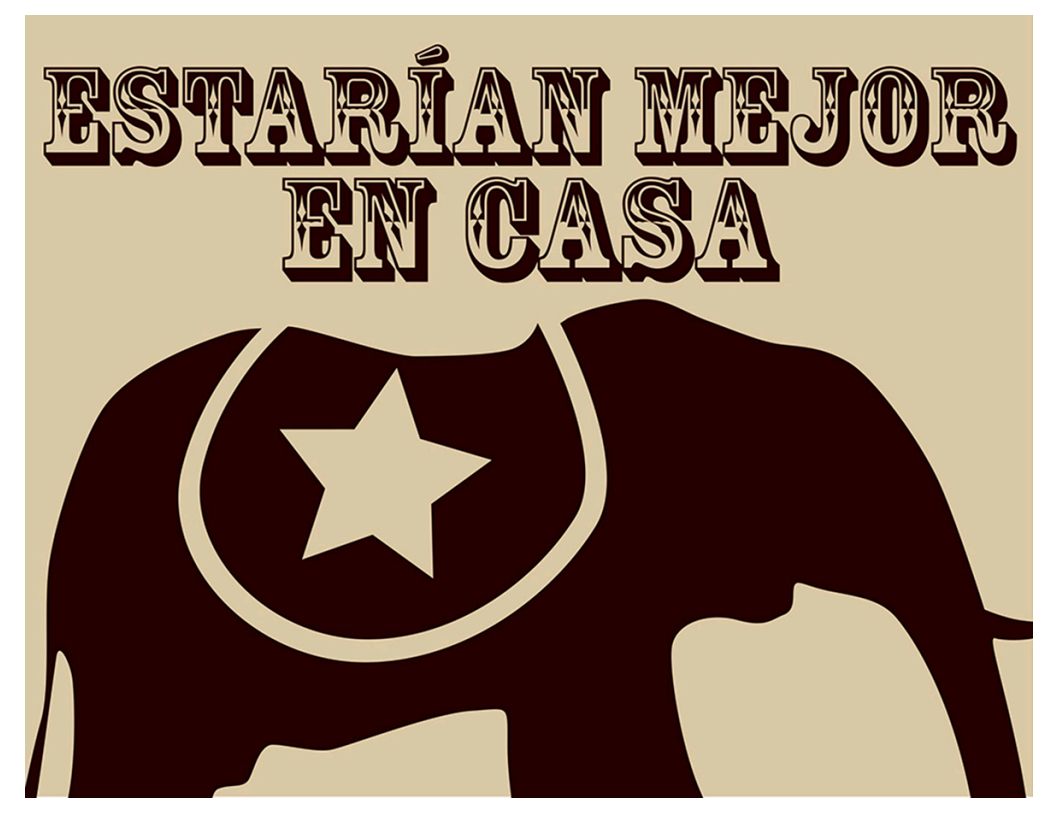

Fig.2 Protección animal - Isabela Muñiz

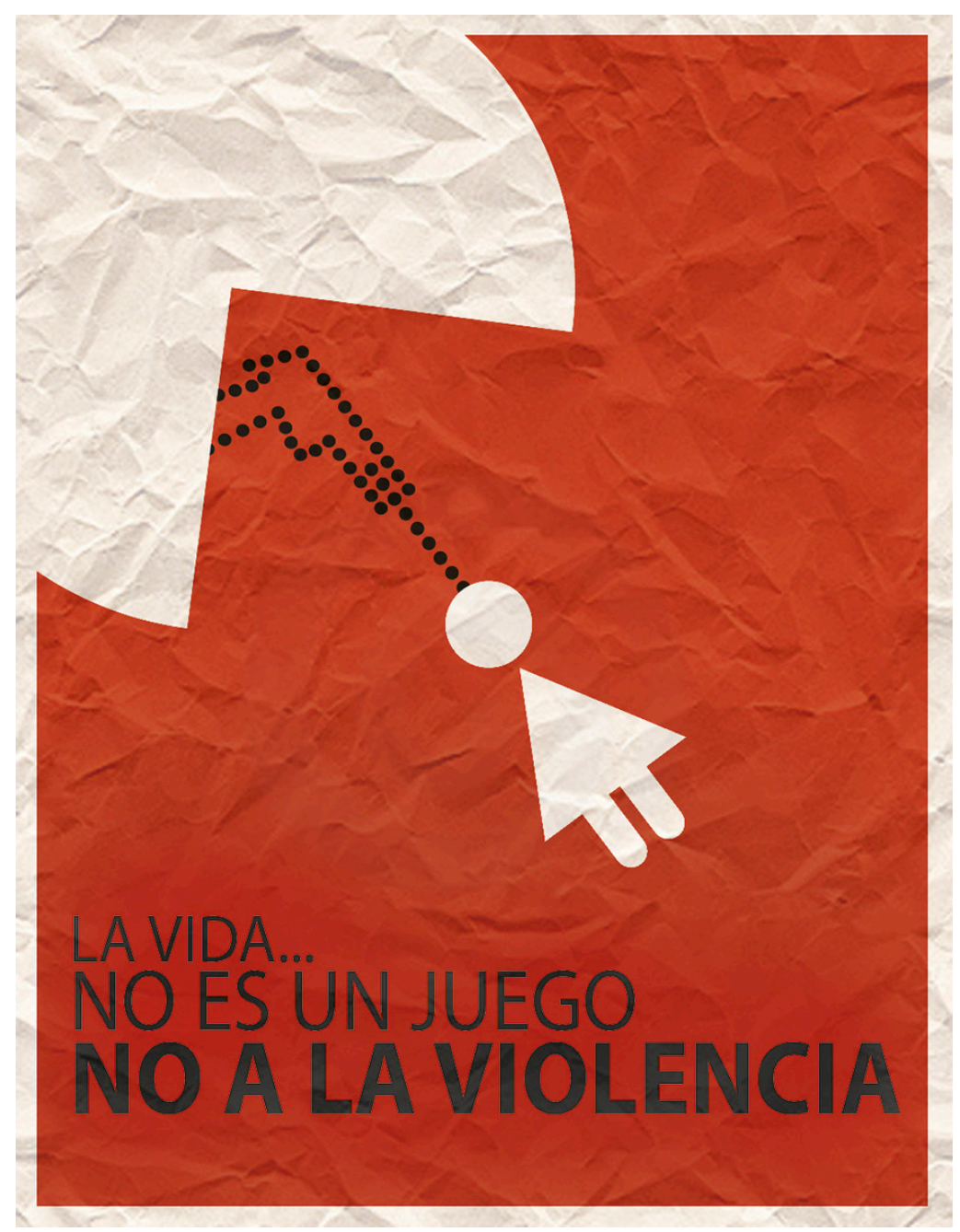

Fig.3 Violencia física y psicológica - Paula Palafox 

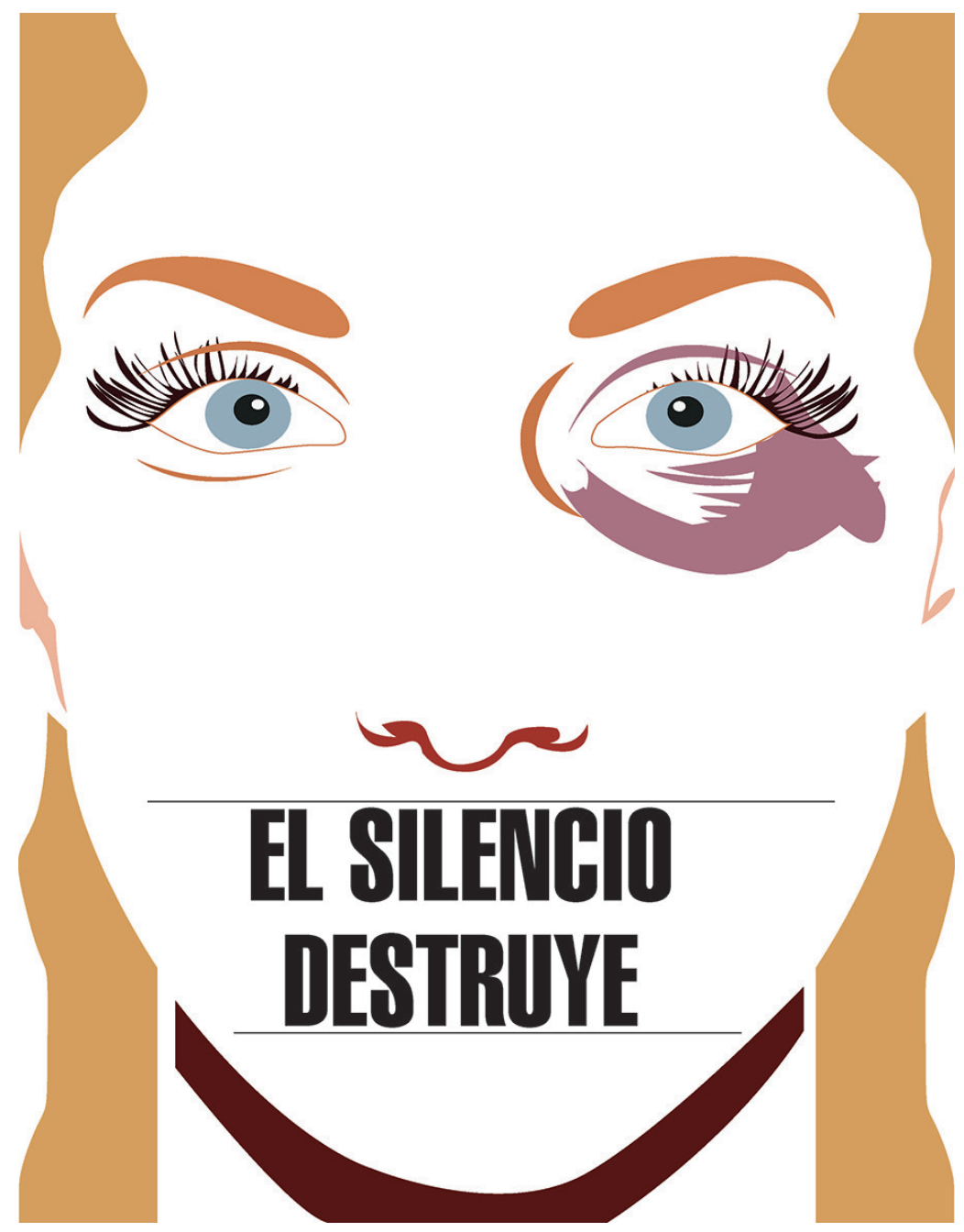

Fig.4 Violencia física y psicológica - Aaron Najera

\section{SOMOS LO MISMO}
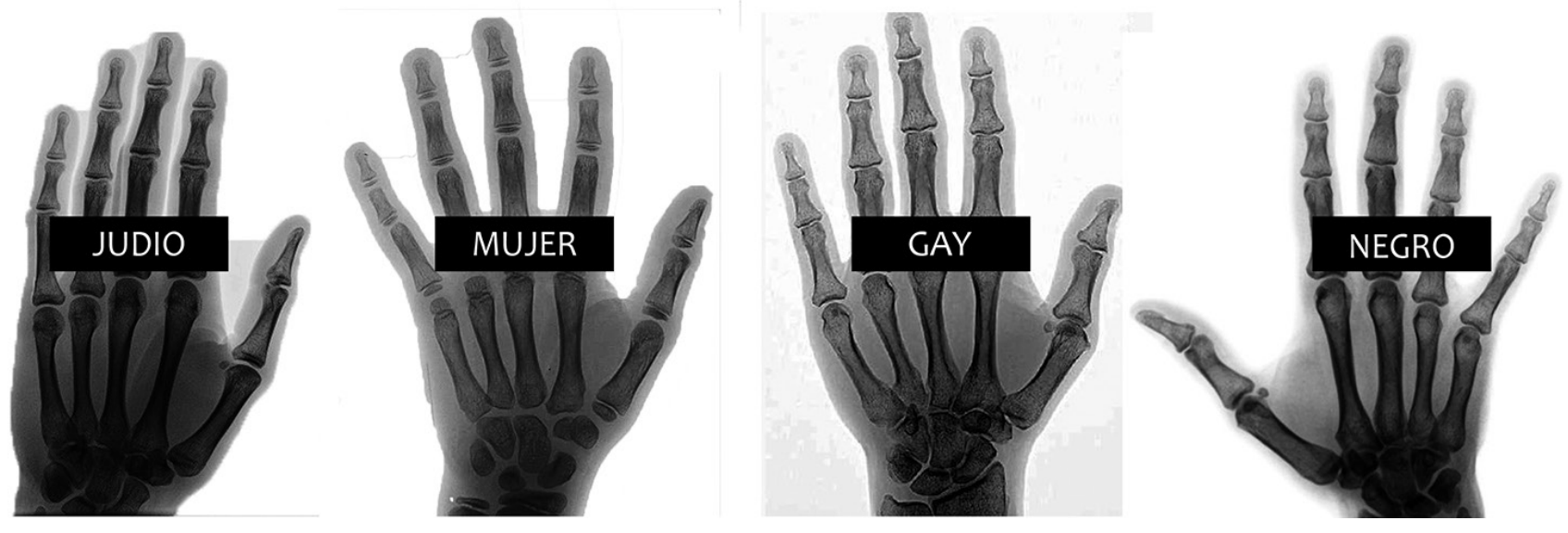

Fig.5 Justicia e Igualdad social - Erik Garcia 


\section{Mejor dos papás...}
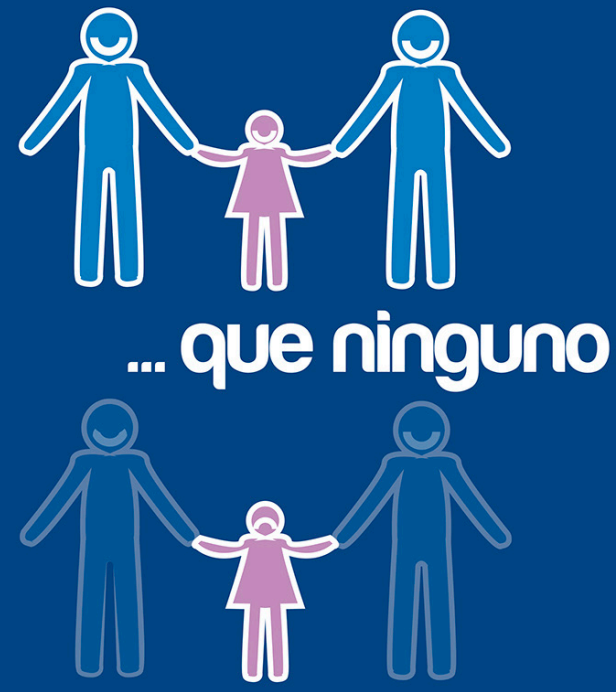

En defensa a la adopción homoparental

Fig.6 Justicia e Igualdad social - David Alvarado

CUIDA EL PLANETA,

ES TU JOYA MAS VALIOSA

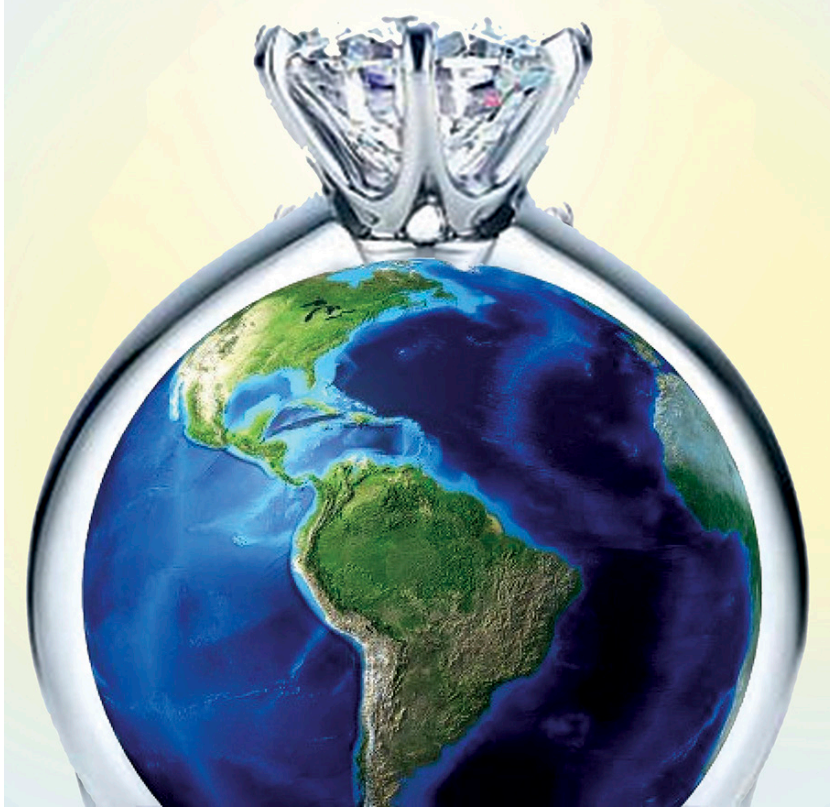

Fig.7 Medio ambiente - Adriana Granados 


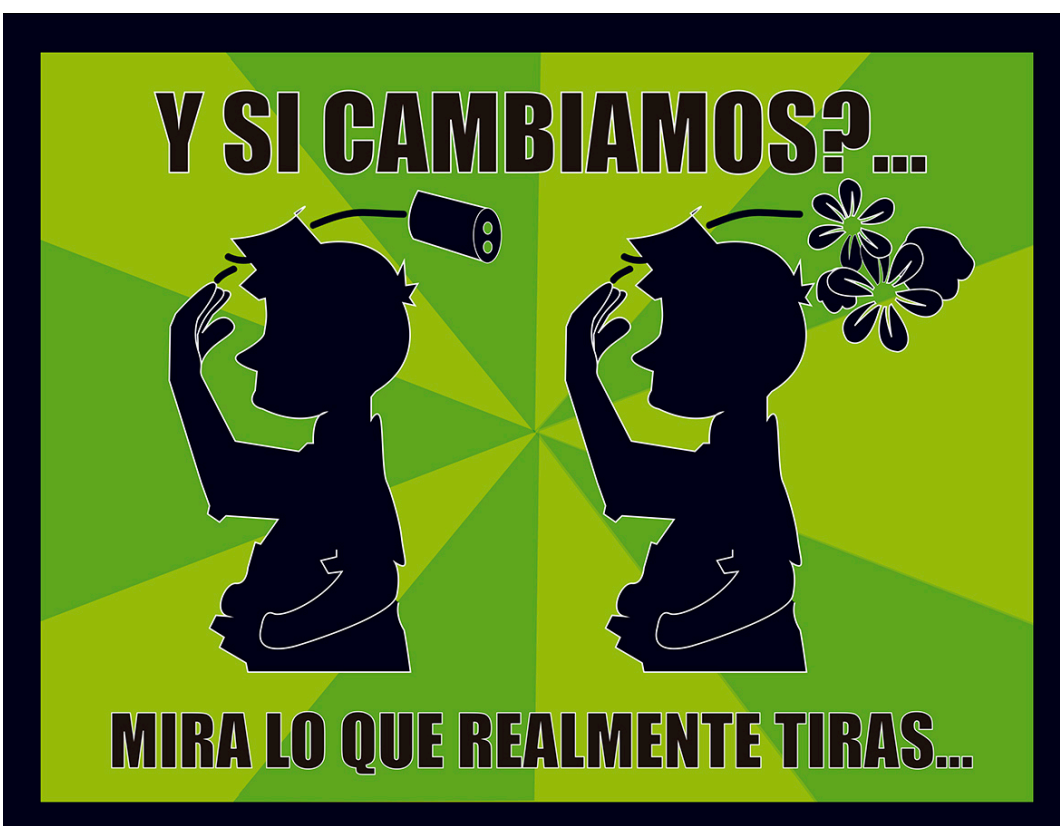

Fig.8 Medio ambiente - Yaneth Morales

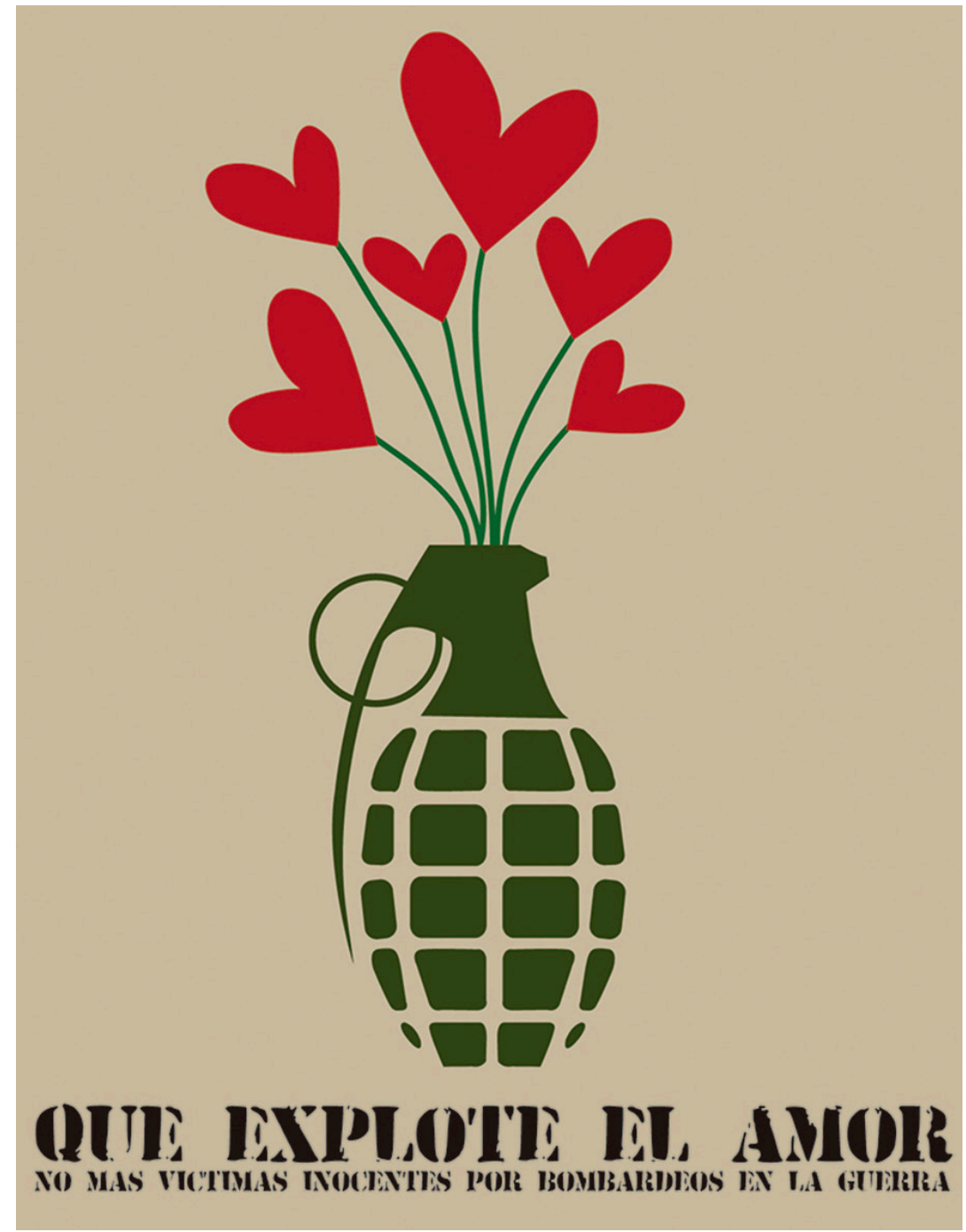

Fig.9 La paz - Kenia Soto 


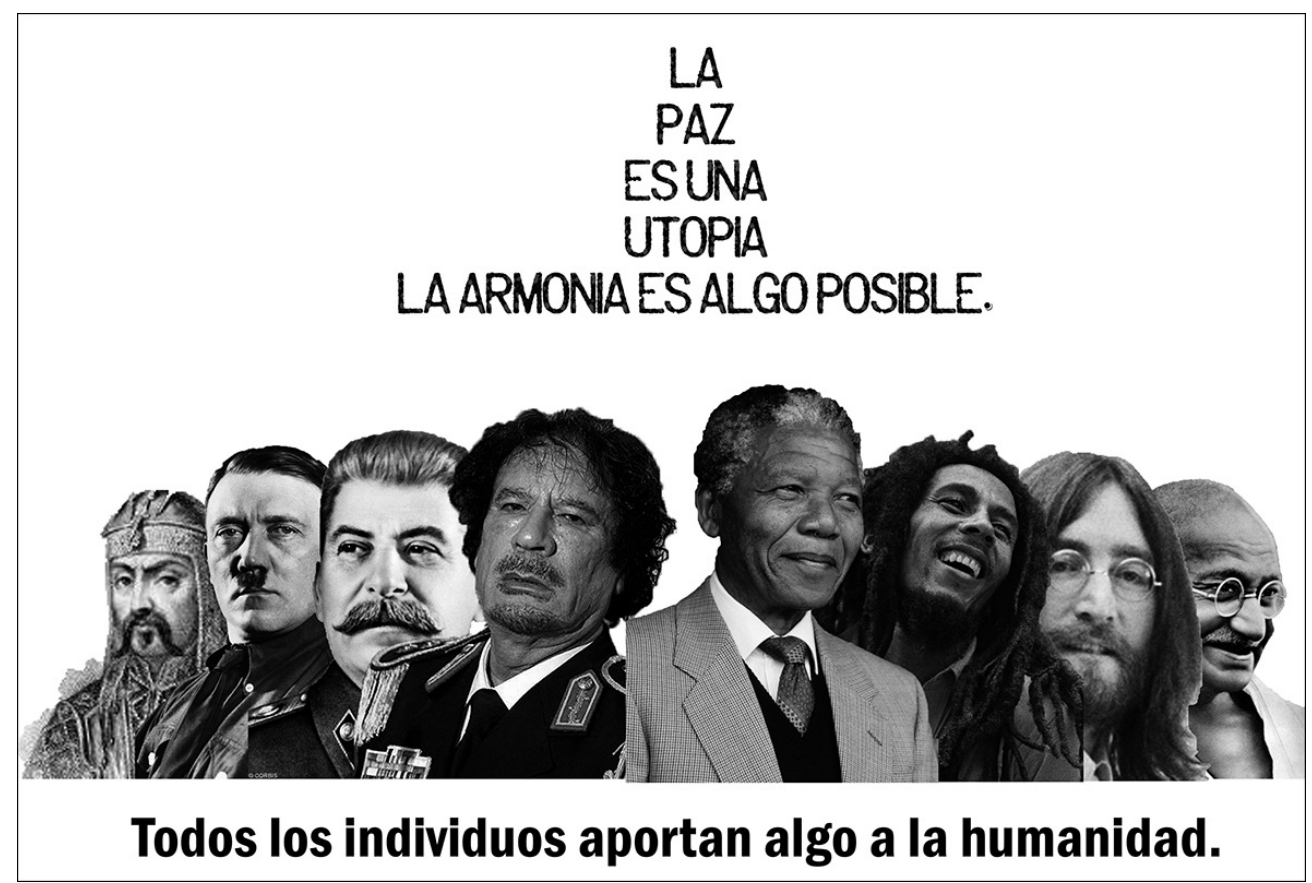

Fig.10 La paz - Josue Flores

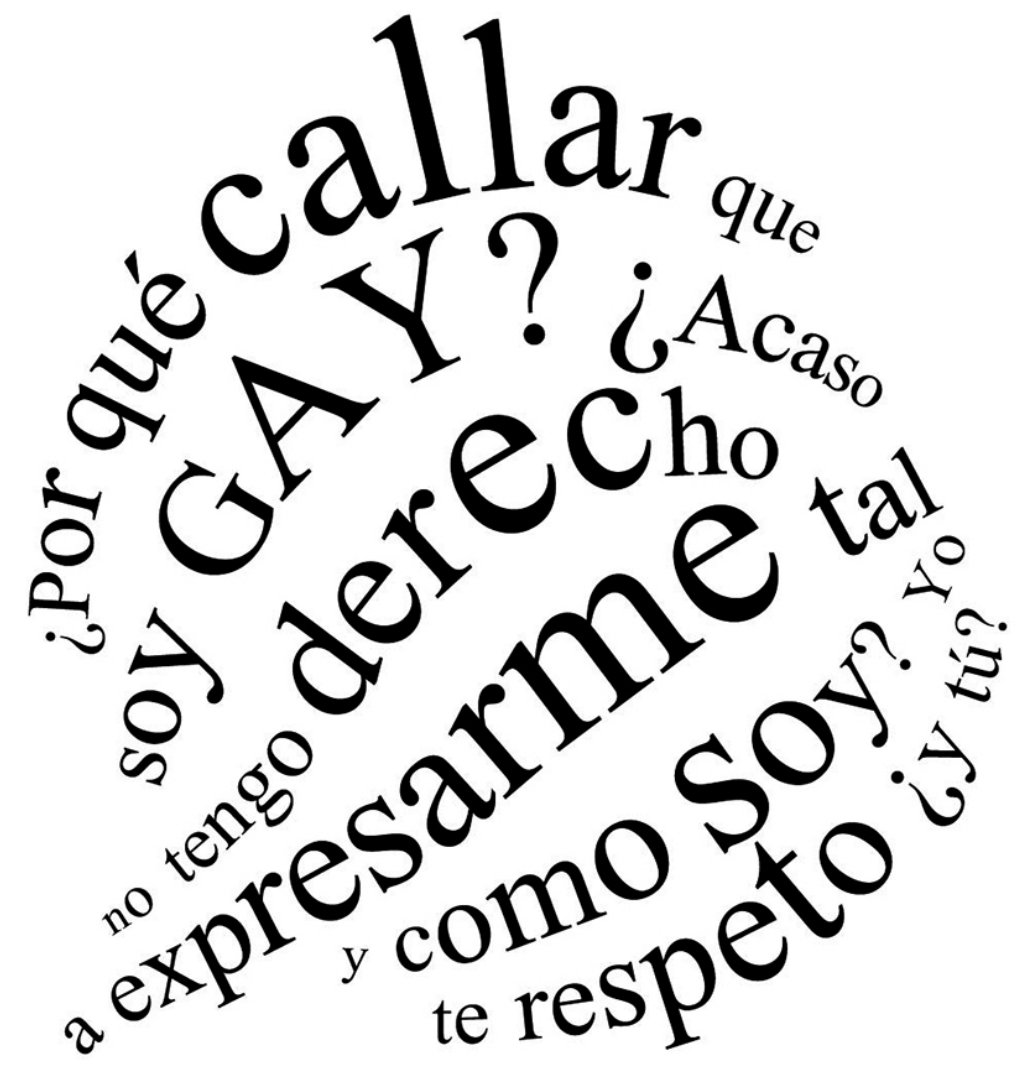

Fig.11 Liberta de expresión y censura - Daniel Balderas 


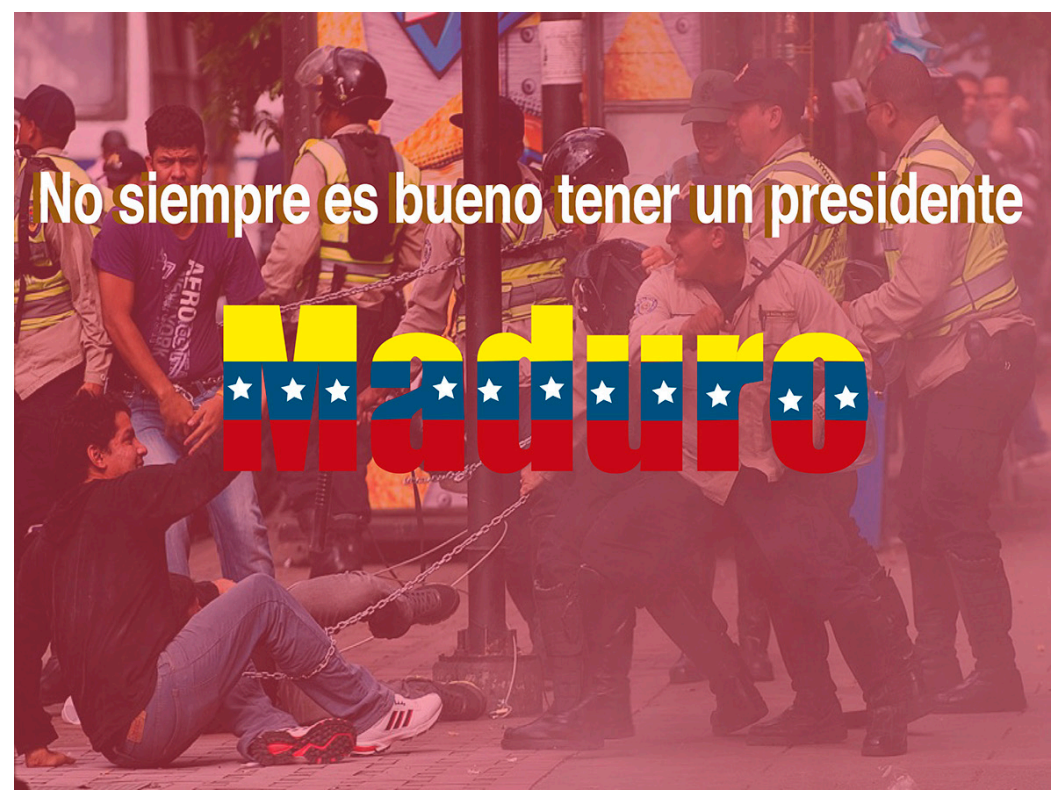

Fig.12 Liberta de expresión y censura - David Alvarado

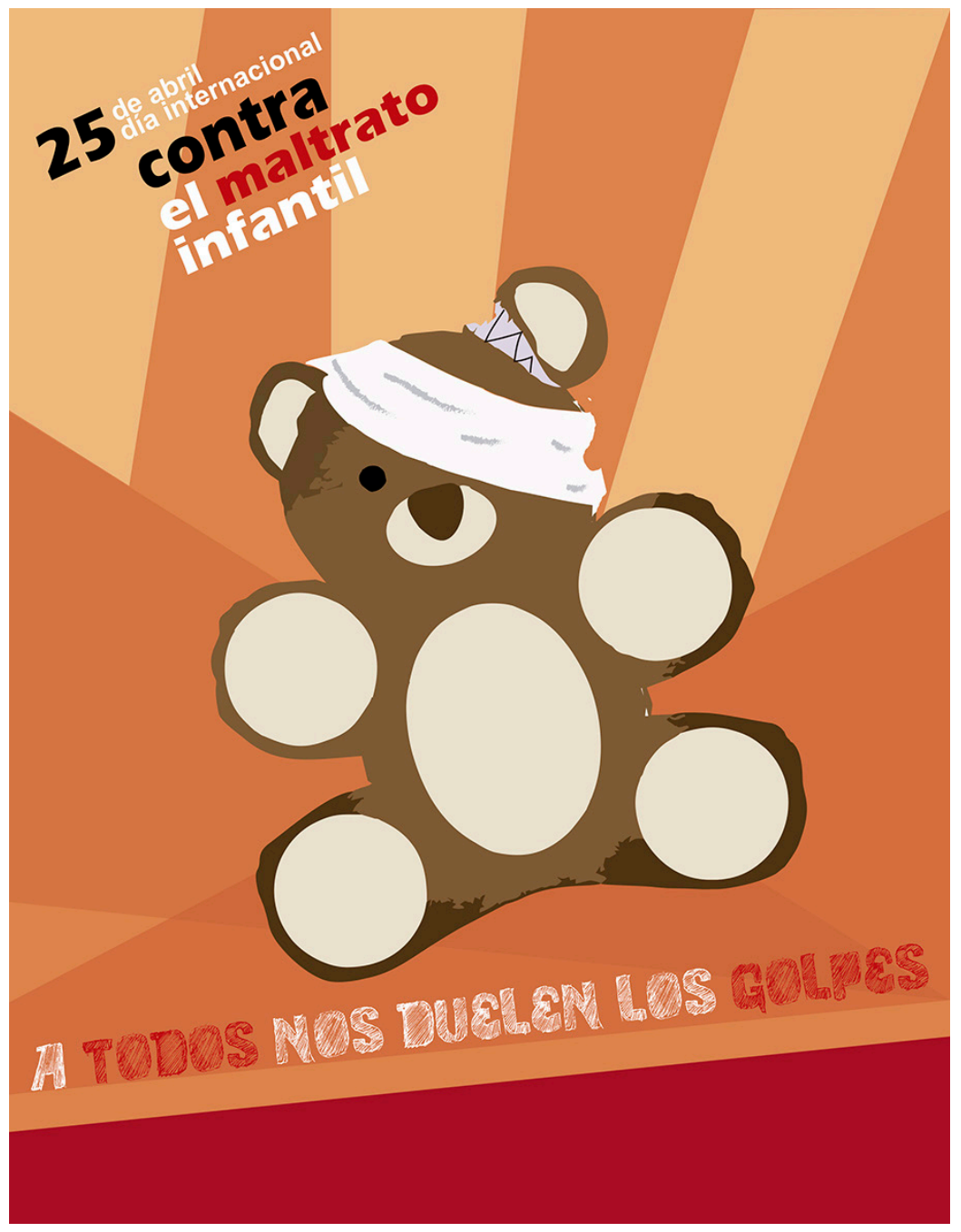

Fig.13 Defensa de la infancia - Omar Jímenez 


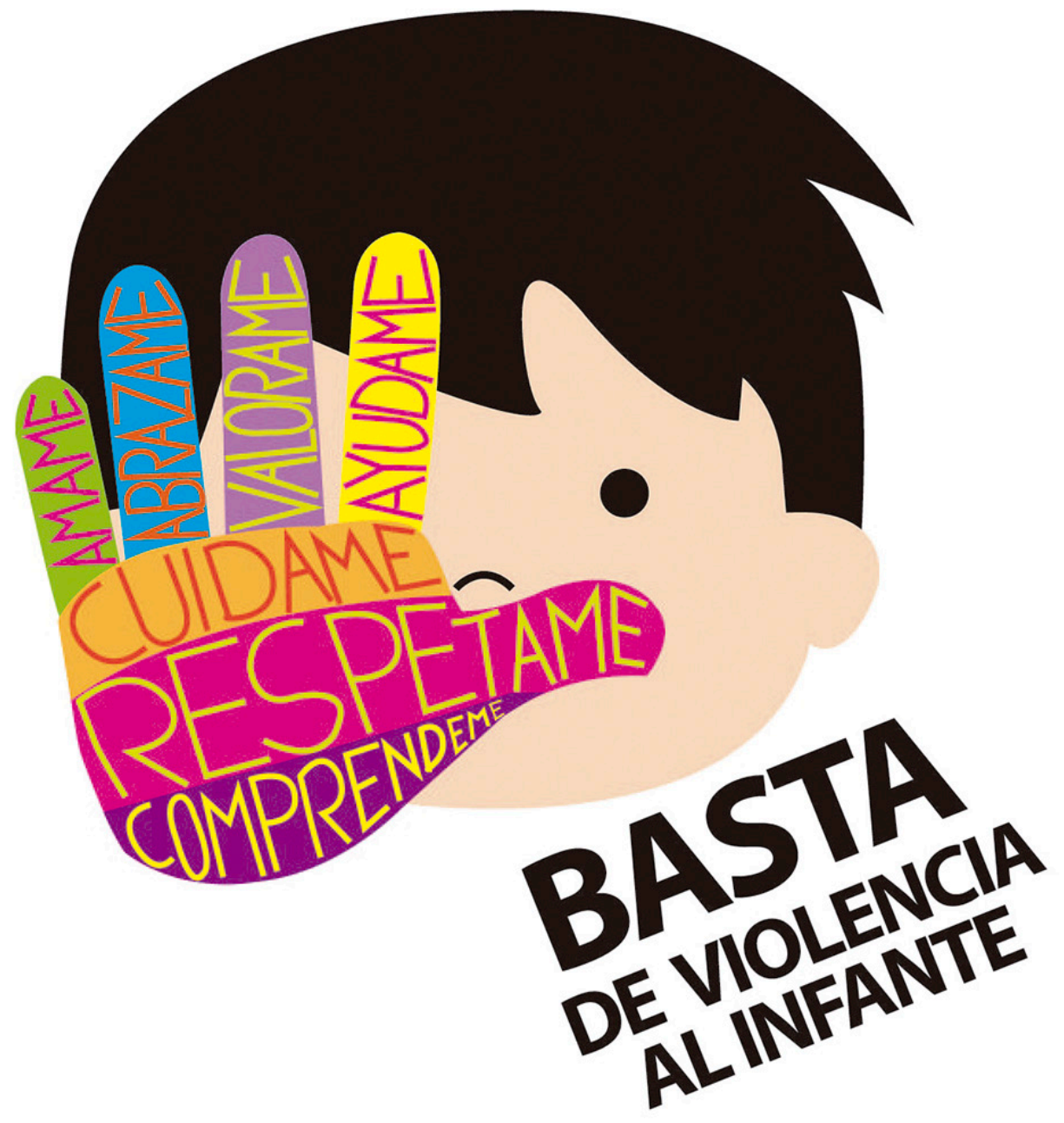

Fig.14 Defensa de la infancia - Karina Hernández

Conclusiones Parece ser que hemos olvidado que no somos sólo técnica y conocimiento. En la educación del alumno se han descuidado las experiencias que propician el desarrollo humano. Se han privilegiado los aspectos del saber y del hacer sobre los del convivir. Se ha subestimado el contacto con los problemas comunitarios, minimizándose estas experiencias como oportunidades, de las que se pueden obtener ideas para la formación de los estudiantes, que serán determinantes en su actuar profesional.

La educación universitaria en su mayoría, centra su interés en elevar indicadores de desarrollo del conocimiento técnico, haciendo poco por desarrollar el aspecto humano de las personas, y decimos en su mayoría, porque consideramos que de 
estar verdaderamente haciendo algo por los aspectos humanos y sociales, el entorno seguramente sería otro.

Es así que, con lo dicho hasta aquí, confiamos en la educación como medio para formar alumnos que en conjunto con los planteamientos escolares formen la "actitud" de trabajar por su entorno social, en aras de contribuir a una mejoría de la realidad al trabajar con planteamientos escolares que tienen que ver con ella. Cuando hay que resolver problemas reales y no hacer que los alumnos trabajen con suposiciones.

Lo dicho permite suponer que los temas que trabaja el activismo gráfico: protección animal, violencia física y psicológica, justicia y equidad social, protección del medio ambiente, paz, libertad de expresión, censura y defensa de la infancia dan la posibilidad de vivir nuevas experiencias que dispongan al alumno a modificar su actitud frente al entorno social, dejando de ser indiferente para convertirse en un ente proactivo y participativo.

La formación universitaria basada en una relación directa con la realidad y sus problemas permite formar "actitudes" en los estudiantes, que, a su vez, determinarán sus vidas, siendo así que las experiencias que vivan formarán nuevas actitudes, sin embargo, las actitudes ya aprendidas (negativas, desinterés, etc.) pueden cambiarse o modificarse con nuevas experiencias. El cambio de actitud tiene su origen en un nuevo aprendizaje, el cual debe ser reforzado para alcanzar el cambio. Mc Guire considera "al refuerzo como un factor crítico en el proceso de cambio actitudinal. Si el individuo recibe refuerzo, la respuesta se fortalece; si por el contrario no se recibe ese refuerzo o se castiga, la respuesta se extingue" (Salazar et al., 2003:171) haciéndose indiferente e insensible.

Recomendaciones

El diseño gráfico puede presentar distintas facetas de proyección separadas de lo comercial. Una de ellas y tal vez la más alejada, es el activismo gráfico. Esta cara del diseño da prioridad al lado humano y pensante del diseñador. Su propósito es transformar la consciencia de las personas, sin embargo, no a todos los diseñadores les interesa realizar ese trabajo ya que por lo general se lleva a cabo sin remuneración y se mantiene en el anonimato. El diseño no comercial es todo un desafío. Algunos consideran que el diseñador debe participar en trabajos sin fines comerciales para lograr un verdadero desarrollo creativo.

Cabe recordar lo expresado acerca de la poca sensibilidad sobre los problemas del entorno social por parte de los individuos que ingresan al sistema de educación superior, por lo tanto, una sugerencia de relevancia mayor es que en cada oportunidad los profesores detecten e integren las dificultades reales del entorno regional haciendo de éstas una experiencia de aprendizaje en el alumno, a fin de iniciar el desarrollo de una actitud de consciencia y responsabilidad social. Ello supone también el compromiso del profesor para estar al tanto, localizar y motivar en el alumno la detección de problemas con la intención de que participe en ellos.() 
Referencias Guzmán, A., (2014). Diseño activista. Tesis doctoral. Facultad de Arquitectura de la Universidad Autónoma del Estado de Morelos. Cuernavaca, Morelos.

Triandis, H. (1971). Attitude and Attitude Change. John Wiley \& sons, Inc.Ovejero, A. (1975). La teoría de la disonancia cognoscitiva. Extracto a partir de la obra de L. Festinger: Teoría de la disonancia cognoscitiva. Madrid: Instituto de Estudios Políticos.

Salazar, J., Montero, M., Muñoz, C., Sánchez, E., Santoro, E., Villegas, J. (2003). Psicología social. México D.F. Trillas/Universidad Central de Venezuela.

Warren, C. (edi.) (1979). Diccionario de psicología. México., FCE. P.4Hernández, R., Fernández, C., Baptista, P. (1998). Metodología de la investigación. México., Mc Graw Hill.

\section{Sobre los autores Guzmán Lechuga Adolfo}

Dr. en Arquitectura, Diseño y Urbanismo por la UAEM., Maestro en Artes Visuales, Comunicación y Diseño Gráfico por la Academia de San Carlos - UNAM y Lic., en Diseño Gráfico por la FAD-UNAM.

Miembro del cuerpo académico Expresión Visual de la Escuela de Artes Plásticas "Rubén Herrera" de la UA de C. Trabaja las líneas de investigación: Imagen, comunicación y cultura, además de Tecnología y procesos creativos del discurso visual. Es profesor en la misma escuela e imparte las materias de Tipografía y los Talleres de Investigación y Producción I y II.

\section{Valdez Borroel María del Socorro Gabriela}

Maestra en Metodología de la investigación por la UA de C y Lic., en Diseño de la Comunicación Gráfica por la FAD-UNAM.

Miembro del cuerpo académico Expresión Visual de la Escuela de Artes Plásticas "Rubén Herrera" de la UA de C. Trabaja las líneas de investigación: Imagen, comunicación y cultura, así como Tecnología y procesos creativos del discurso visual. Es profesora en la misma escuela e imparte las materias de Teoría y Percepción del Color, Envase y Embalaje, además de Factores Económicos para el Diseño. 